\title{
MALABSORBTION AND SMALL INTESTINAL MORPHOLOGY IN INFECTIVE HEPATITIS
}

\author{
Lieutenant-Colonel I. MICHIE, M.B., M.R.C.P.(Ed.), R.A.M.C \\ Military Hospital, Tidworth
}

SUMMARY: The intestinal malabsorption pattern of 50 male patients suffering from infective hepatitis was studied. A total of 78 per cent had faecal fat excretion of greater than seven grams per day, while the D Xylose absorption was normal. There was no correlation between the severity of the clinical illness, serum levels of bilirubin, alkaline phosphatase or transaminases and the steatorrhoea. Jejunal mucosal biopsies from a site immediately distal to the duodeno-jejunal flexure showed only three with abnormal changes. This finding shows that there is no characteristic jejunal mucosal change in infective hepatitis and that the steatorrhoea is unrelated to intestinal mucosal change.

Introduction

The cause of steatorrhoea in infective hepatitis is still uncertain and a number of mechanisms have been evoked. Astaldi, Strosseli, Poggi and Grandini (1963 \& 1964) showed marked intestinal mucosal changes in patients with infective hepatitis and these changes were suggested as a contributing cause of the steatorrhoea found in this condition. These findings were confirmed by other workers (Conrad 1966), (Conrad, Schwartz and Young 1964), (Sheehy, Artenstein and Green 1964) but in a recent study (Kudzma, Peterson and Knudson 1969) no intestinal mucosal changes were found in 18 cases of infective hepatitis.

Colwell (1957) and Gross (1950) found that the faecal fat excretion was greatest when the jaundice was most severe, suggesting that a decrease of bile reaching the intestine might be important in the production of the steatorrhoea.

In order therefore to clarify these findings a study of the mucosal lesions and the absorptive capacity of the intestine was undertaken in 50 patients with infective hepatitis.

\section{Materials and methods}

Fifty male patients of European stock, admitted consecutively to the Military Hospital, Terendak, Malaysia were the subjects for this study. The age of the patients ranged from seven to 39 years (Mean 24 years) and all except two, aged seven and 12 years were serving adult military personnel. Two patients had been in the tropics for less than one month; 21 for less than one year; 15 for less than two years and 16 for a longer period. The patients were treated in a general hospital ward, allowed up for toilet purposes only and were given a normal hospital diet. No drugs were administered.

The diagnosis of infective hepatitis was made from the clinical presentation of the illness, the serum direct and indirect bilirubin, serum transaminases and the serum alkaline phosphatase levels. The diagnosis was confirmed by liver biopsy in two instances. The Paul Bunnell screening test for infective mononucleosis was positive in one patient, while the toxoplasma dye test was above 1:64 in three, two with a titre of $1: 256$ and the other 1:512. There was no significant difference between these patients and the remainder, either in presentation, course or duration of their illness. 
Blood was taken on admission for haemoglobin, white cell count, erythrocyte sedimentation rate and liver function tests. Three day stools were collected and the daily faecal fat excretion estimated. (Kamer, Bokkel Huinink and Weyers 1949). A five gram D Xylose absorption test was also performed. (Santini, Sheehy and Martinez-De-Jesus 1961).

An intestinal biopsy was performed at a site immediately distal to the duodenojejunal flexure using the Watson capsule. The jejunal mucosa was examined initially under the dissecting microscope and subsequently with the conventional light microscope. The sections which were stained with haematoxylin and eosin were reviewed by two independent observers.

\section{Results}

The faecal fat was greater than seven grams per day in 39 of the 50 patients, the mean daily excretion being $13.1 \mathrm{~g}$ per day. In 36 of the cases the faecal fat was measured during the first week of illness and during this period 24 of the 36 patients excreted more than seven grams per day. The mean excretion rate during this initial period was $11.5 \mathrm{~g}$ per day.

Forty-one patients had a D Xylose absorption test on one or more occasions. The urinary excretion of Xylose was less than $1.2 \mathrm{~g}$ on only four occasions and in none of these four did the excretion fall below one gram per day. The mean daily faecal fat excretion of these four patients with low urinary excretion of Xylose was $16.9 \mathrm{~g}$ per day.

The relationship between the serum level of bilirubin and the faecal fat excretion was closely studied. Figure 1 illustrates the finding that there is no correlation between the amount of fat excreted and the serum level of bilirubin. The serum levels of alkaline phosphatase and transaminases were plotted in a similar manner but again no correlation was seen between the amount of fat excreted and these indices of liver function.

A jejunal biopsy was obtained in 38 of the 50 patients studied: all but eight were obtained in the first two weeks of their illness. Under the dissecting miscroscope 36 of the 38 biopsies showed a normal mucosal pattern while the remaining two showed only mild abnormalities. These two biopsies showed predominately broad leaves joining together in places to form ridges. On light microscopy one of these two biopsies and one additional biopsy showed some blunting and widening of the villi. The remaining 36 showed a normal jejunal mucosal architecture and careful study of the mucosa compared with normal tissue showed no increase in cellular infiltration. There were therefore three mildly abnormal biopsies by both dissecting microscope and histological criteria. There was no significant relationship between these biopsy findings and the severity of illness or the degree of steatorrhoea.

\section{Discussion}

The pattern of intestinal malabsorption demonstrated by these patients is one of steatorrhoea with normal Xylose absorption and no significant jejunal mucosal abnormality. The incidence of steatorrhoea was 78 per cent in this group taking the normal level of excretion as seven grams per day; this figure is similar to other published reports (Kamer, Bokkel Huinink and Weyers 1949).

The subjects in this study did not differ significantly from those of Conrad, Schwartz and Young (1964) who described the villi as being shortened and club shaped. In addition 


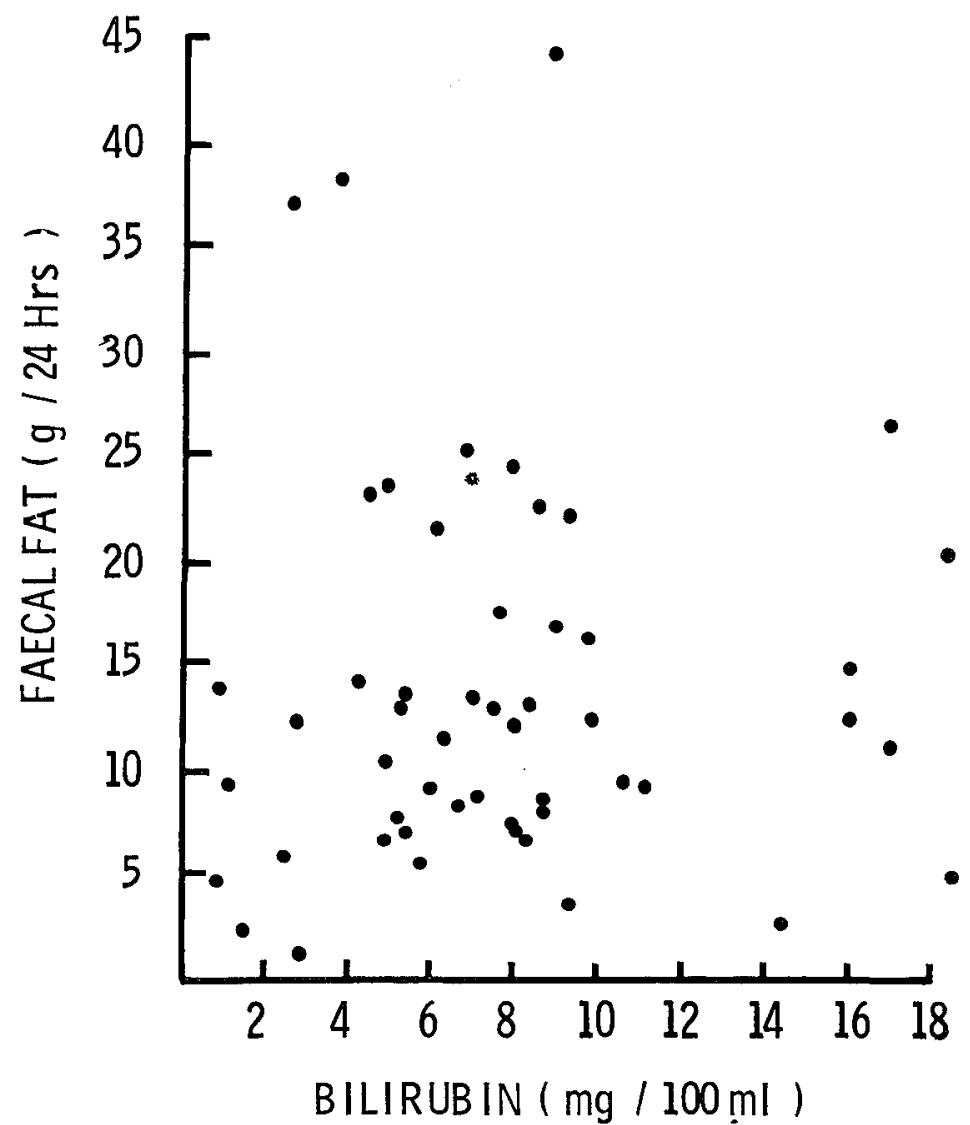

Fig. 1. The relationship between bilirubin $(\mathrm{mg}$ per $100 \mathrm{ml})$ and daily fat excretion ( $g$ per day).

these workers found some patchy lesions with complete absence of villi and most of their biopsy specimens showed infiltration of the lamina propria with lymphocytes and plasma cells. Some of their mild mucosal abnormalities may be explained on the normal variation found in people living in Asiatic countries (England and O'Brien 1966), (Lindenbaum, Alam and Kent 1966); however it does not explain the subtotal villous atrophy seen in at least some of their cases.

The changes found by Sheehy, Artenstein and Green (1964) in six American soldiers with infective hepatitis were again variable, consisting mainly of blunting and fusion of the villi with some cellular infiltrate. These workers found no similar abnormality in patients with infective mononucleosis and they proposed that small intestinal biopsy may be a way of differentiating the two diseases. The mucosal changes were however only seen to any degree in three of their six patients and from our data abnormal jejunal :mucosa must be an unusual occurrence in infective hepatitis.

Kudzma, Peterson and Knudsen (1969) contrary to the above reports found normal jejunal biopsies in their cases agreeing with the findings reported here. To account for the difference between their findings and earlier studies, they suggest regional variation, the degree with which different hepatitis viruses affect the gut and finally the orientation 
of the biopsy specimen. Current orientation of the biopsy specimen and careful attention to histological detail is essential to exclude artefacts (Rubin and Dobbins 1965) and it is probable that most of the results in earlier reports can be explained on this basis. We cannot however exclude the possibility that mucosal abnormalities do occur in some cases in infective hepatitis but this report together with that of Kudzma, Peterson and Knudsen (1969) must suggest that significant abnormality is a rare occurrence.

Steatorrhoea is a prominent feature of infective hepatitis and a mucosal abnormality is not the cause. In addition to the normal intestinal biopsy, the absorption of Xylose was normal compared to a similar group of subjects recently studied (Keusch, Plaut and Troncale 1970). The small intestine thus seems to be normal in infective hepatitis. What then is the cause of the steatorrhoea?. Gross (1950) studied pancreatic secretion after a dose of secretin and found no decrease in bicarbonate output in his patients. In our series as well as that reported by Conrad (1966) there was no correlation between the steatorrhoea and the serum levels of bilirubin, alkaline phosphates or transaminases. In addition Gross (1950) could find no quantitative deficiency of bile in his patients with infective hepatitis and steatorrhoea. That the severity of the hepatic disease and the degree of cholestasis are unrelated to the daily faecal fat excretion would with the above finding suggest that lack of bile reaching the intestine is not the whole answer. It is however quite possible that the lack is qualitative rather than quantitative and bile salt deficiency may exist.

In conclusion it may be said that the cause of steatorrhoea in infective hepatitis has not been established. This report confirms another recent study that there is no characteristic abnormality of the jejunal mucosa in infective hepatitis.

\section{REFERENCES}

Astaldi, G., Strosseli, E., Poggi, C. and Grandini, U. (1963). Boll. Soc. ital Biol. sper. $39,589$. Astaldi, G., Grandini, U., Poggi, C. and Strosseli, E. (1964). Amer. J. dig. Dis. 9, 237.

Colwhell, A. R. (1957). Gastroenterology. 33, 591 .

ConRaD, M. E. (1966). J. chron. Dis. 19, 199.

Conrad, M. E., Schwartz, F. D. and Joung, A. A. (1964). Amer. J. Med. 37, 789.

ENGLAND, N. W. and O'BRIEN, W. (1966). Gut 7, 128.

Gross, J. B. (1950). Gastroenterology 16, 140.

KAMER, J. H. van de BoKkel HuININK, H. ten and Weyers, H. A. (1949). J. biol. Chem. $177,347$.

Keusch, G. T., Plaut, A. G. and Troncale, J. (1970). Lab. Clin. Med. 75, 558.

Kudzma, D. J., Peterson, E. W. and Knudsen, K. B. (1969). Arch. intern. med. 124, 322.

Lindenbaum, J., AlaM, A. K. M. M. and Kent, T. H. (1966). Brit. med. J. ii, 1616.

RubIN, C. E. and DobBINS, W. O. (1965). Gastroenterology 49, 676.

Santani, R., Sheehy, T. W. and Martinez-DE-Jesus, J. (1961). Gastroenterology, 40, 772.

Sheehy, T. W., Artenstein, M. S. and Green, R. W. (1964). J. Amer. med. Ass. 190, 1023. 